\title{
A STUDY OF PROGNOSTIC FACTORS IN CARCINOMA BREAST
}




\section{Sld Bot}


Jemds.com 
Majority of cases showing recurrence had ILC (66.7\%) whereas majority of cases not showing recurrence were IDC (80.9\%). Statistically, there was a significant difference between two groups with respect to FNAC/ biopsy findings ( $p=0.001)$.

Sl. No. Finding $\quad$ Recurrence $(n=12)$ 

Jemds.com 